\title{
Transcatheter Aortic Valve Replacement versus Surgical Aortic Valve Replacement: A Review of Aortic Stenosis Management
}

\author{
Yasar Sattar $^{1}$, Hiba Rauf ${ }^{2}$, Syeda Beenish Bareeqa ${ }^{3}$, Waqas Ullah ${ }^{4}$, Madhura Myla ${ }^{5}$ \\ 1. Internal Medicine, Icahn School of Medicine at Mount Sinai, New York, USA 2. Internal Medicine, Dow Medical \\ College, Karachi, PAK 3. Internal Medicine, Jinnah Medical and Dental College, Karachi, PAK 4. Internal Medicine, \\ Abington Hospital - Jefferson Health, Abington, USA 5. Cardiovascular Disease, University of New Mexico Health \\ Sciences Center, Albuquerque, USA
}

Corresponding author: Yasar Sattar, sattary@nychhc.org

\begin{abstract}
Severe aortic stenosis (AS) affects 3.4\% of the elderly over 60 years of age. It presents with exertional dyspnea, syncope, angina, and progression to irreversible congestive heart failure. Early intervention produces a better outcome in preventing the clinical deterioration of AS. The choice of intervention is transcatheter aortic valve implantation or surgical aortic valve replacement (SAVR). The decision should be made after evaluating an individual case based on its clinical features and the user's experience with transcatheter aortic valve replacement (TAVR). We reviewed available data to illustrate the types of ASs, the background of interventions, current guidelines for TAVR, and its comparison with SAVR in terms of adverse effects.
\end{abstract}

Categories: Cardiac/Thoracic/Vascular Surgery, Cardiology, Internal Medicine Keywords: severe aortic stenosis, transcatheter aortic valve implantation, transcatheter aortic valve replacement (tavr), surgical aortic valve replacement

\section{Introduction And Background}

Aortic stenosis (AS) is defined as left ventricular outflow tract obstruction due to the narrowing of the orifice of the aortic valve with a forward velocity of blood at least $2 \mathrm{~m} / \mathrm{sec}$. In Europe and the United States, calcific AS holds the most share in causing AS in people older than the sixth decade of life, with the congenital bicuspid valve being the most commonly affected among those in their fourth to sixth decade of experience [1]. The pooled estimated prevalence of AS among the elderly is $12.4 \%$ and that of severe AS is $3.4 \%$ [2]. Criteria establish severe AS when transvalvular aortic velocity is $>4 \mathrm{~m} / \mathrm{sec}$ when the aortic jet velocity is $\geqslant 5$ $\mathrm{m} / \mathrm{sec}$, and the aortic orifice is $\leqslant 1 \mathrm{~cm} 2$. Severe AS is a significant area of concern due to the poor prognosis and deterioration of inotropy, thus requiring early intervention. AS can initially present with exertional dizziness, dyspnea, reduced exercise tolerance, and progression to irreversible congestive heart failure, syncope, and angina in severe AS. Therefore, early intervention in severe AS has better clinical outcomes. The choice of the optimal mode of response has been a topic of debate for the past decade, with several trials assessing the efficacy and safety of transcatheter aortic valve implantation/replacement (TAVI/TAVR) versus surgical aortic valve replacement (SAVR). Approximately 67,500 surgeries are performed annually for aortic valve replacement (SAVR) [3]. A recent study performed by Osnabrugge et al. suggests an estimated prevalence of 3.4\% of severe AS in the elderly population in their seventh decade of life. Out of this, approximately 290,000 people over 70 years of age are potentially high risk for surgery that could be managed by TAVR in Europe and North America, with an annual number of 27,000 new candidates for TAVR. This review highlights AS criteria, the background of procedures, current guidelines for TAVR, comparison of different approaches, and future perspectives in the treatment of AS [2,4].

\section{Review}

\section{Literature search strategy}

Relevant medical literature was searched using MEDLINE, Scopus, Ovid SP, and Google scholar. Studies were included based on the availability of full text, literature in the English language, and date published between 2005 and 2019. Studies with inconclusive scientific evidence, literature in a language other than English, and those with unavailable full texts were excluded from this review. The search strategy was based on the combinations of medical subject heading, including " $\mathrm{s}$ ", "Transcatheter Aortic Valve Implantation", "Transcatheter Aortic Valve Replacement", and "Surgical aortic valve replacement".

\section{Aortic stenosis}

The standard aortic orifice is 3.0 to $4.0 \mathrm{~cm} 2$ [5]. AS is specified as the narrowing of the valve orifice and a maximal transvalvular velocity of $\geqslant 2 \mathrm{~m} / \mathrm{sec}$ [1]. The transvalvular pressure gradient through the orifice of the valve reduces as a result of stenosis. As the pressure gradient also depends on transvalvular flow, it is low in 
a decreased cardiac output state. AS is categorized into three types, mild, moderate, and severe, depending on valve orifice size, transvalvular velocity, and pressure. Mild AS has a valve orifice of $>1.5 \mathrm{~cm} 2$, a transvalvular pressure of $<20 \mathrm{~mm} \mathrm{Hg}$, and a flow velocity of 2.5 to $3 \mathrm{~m} / \mathrm{sec}$ [6]. Moderate AS has a valve orifice of 1.0 to $1.5 \mathrm{~cm} 2$ with a transvalvular pressure of 25 to $40 \mathrm{mmHg}$ when the transvalvular flow is at baseline [4]. Severe AS has a valve orifice of $<1 \mathrm{~cm} 2$, a transvalvular pressure of $>40 \mathrm{mmHg}$, and a flow velocity of $>4 \mathrm{~m} / \mathrm{sec}$ [3]. The criteria for severe AS have high sensitivity due to a broad basis for inclusion, and therefore many patients who fall in this category are asymptomatic or have mild symptoms. These patients require early intervention as they are at an increased risk of sudden death and have a maximum survival of two to three years [7].

\section{History}

Over the decades, SAVR with a mechanical or bioprosthetic valve has been the cornerstone for the management of severe cases of AS to improve heart function and survival [4,8]. In 1980, H.R. Anderson tested a balloon-expandable valve in animals followed by Alain Cribier, who proposed the transfemoral arterial approach in humans in 2000. Since then, many renowned scientists have worked on different approaches until Edwards Lifesciences identified a flexible catheter that passed across the aortic arch through the retrograde transfemoral approach. With continuing advances, Medtronic (Minneapolis, MN) introduced a nitinol-based self-expandable valve called CoreValve. The effectiveness of the Edwards SAPIEN (Edwards Lifesciences, Irvine, CA) and Medtronic CoreValve was proven by implantation of these valves at the aortic valve using the transcatheter approach in the PARTNER (Placement of Aortic Transcatheter Valves) trial and the United States CoreValve Pivotal Trial, respectively. At present, both valves are approved by the U.S. Food and Drug Administration (FDA) and employed in treatment by different institutes [9].

\section{Medical therapy}

Medical therapy for AS has not shown any promising results in establishing the efficacy of statins, antihypertensive drugs, and drugs that target phosphate and calcium for AS. However, future research may reveal minimized left ventricle (LV) remodeling in AS through angiotensin-converting enzyme inhibitors, angiotensin-receptor blockers, and sacubitril as post-aortic valve replacement medical therapy $[4,8,10]$.

\section{Current indications for intervention in aortic stenosis}

Patients with AS can be categorized as symptomatic or asymptomatic. The decision and choice of intervention depend on symptom severity and associated comorbidities. According to the 2017 European Society of Cardiology guidelines, intervention is indicated in all symptomatic patients with severe high gradient AS. Intervention should also be performed in symptomatic patients with severe low-flow, lowgradient AS with reduced ejection fraction. However, when the intervention does not seem to provide any improvement in the quality of life or survival due to debilitating comorbidities, balloon dilation can be considered [11]. All asymptomatic patients who have severe AS with abnormal exercise tolerance tests or reduced ejection fraction should also undergo intervention [4]. The intervention should be performed in a facility that has a cardiology department, cardiac surgery department, and a heart team consisting of an interventional cardiologist, imaging specialists, cardiothoracic surgeons, cardiac anesthetists, and nurses [12].

\section{Symptomatic patients}

The decision between SAVR and TAVI should be based on individual patient preference and weighing of the risk-benefit ratio. Before deciding on the type of procedure, the surgical risk score should be determined using the Society of Thoracic Surgery (STS) score or EuroSCORE (European System for Cardiac Operative Risk Evaluation) $[9,12]$. These scores give the percentage of predictable poor outcomes based on a patient's clinical features. If the patient is deemed to be at a low surgical risk (the STS score or EuroSCORE II is $<4 \%$ ), SAVR can be safely performed in symptomatic AS patients. However, if the STS score or EuroSCORE II is $>4 \%$, then the multidisciplinary heart team should account for individual patient features. In these cases, TAVI can be considered as a suitable intervention. Another procedure, balloon aortic valvotomy, is a diagnostic and therapeutic intervention. It can be performed as bridge therapy in hemodynamically unstable patients or severe AS patients who urgently need major noncardiac surgery. Later, the patient can undergo SAVR or TAVI when stabilized [4].

\section{Asymptomatic patients}

Firstly, the patient should be assessed for symptoms such as exertional dyspnea, chest pain, and dizziness. If the patient has equivocal symptoms, then an exercise stress test should be employed [13]. As TAVI is not recommended in asymptomatic patients with severe AS, SAVR can be performed as an elective procedure in those who are prone to become symptomatic if left untreated $[4,10]$. The rate of event-free survival at two years is only $30 \%$ to $50 \%$; therefore, the importance of serial follow-up to monitor progression should be emphasized [14]. The follow-up investigations can include performance on the exercise stress test, changes in echocardiographic findings, and measurement of brain natriuretic peptide [4]. In a multicentric trial, mortality was assessed in asymptomatic severe stenotic patients who either received early surgery or conservative treatment. The study showed significantly lower mortality rates from cardiovascular causes in 
the early SAVR group [15].

\section{SAVR procedure}

SAVR is an extensive heart surgery performed under general anesthesia on a cardiopulmonary bypass. A median incision is made on the sternum to expose the mediastinum. It is followed by dissecting the aorta and removing the stenotic valves. Finally, the native valves are replaced with either mechanical or bioprosthetic valves [16]. After the surgery, the patient is generally kept in the intensive care unit (ICU) for 24 to 48 hours and for a week in the inpatient unit to monitor for complications.

\section{TAVI pre-procedural evaluation}

Transthoracic echocardiography is used to assess the degree of AS, valve calcification, the severity of left ventricular dysfunction, and accompanying valvular abnormalities [4]. If this modality is not sufficient to make the diagnosis, transesophageal echocardiography is used to define the valvular anatomy and caliber further [12]. Nowadays, a computerized tomography (CT) scan is preferred as the diagnostic modality due to its accuracy and precision. The diagnostic evaluation can help the heart team to decide on the dimensions of the prosthetic valve, mode, and route of the intervention [17].

\section{Implantation approaches}

TAVI can be performed through several routes, most importantly, transfemoral, transaortic, and transapical. The transfemoral route is considered the default approach with better results than the transapical route [18]. Other routes that can be used in cases of difficult femoral access are transcarotid, transaxillary, and transvenous routes [19].

\section{Anesthesia}

TAVR can be performed with the patient under local anesthesia with moderate sedation or awake, invariable general anesthesia, and complete general anesthesia for transfemoral, transapical, and transaortic approaches, respectively [20]. The awake TAVR is considered monitored anesthesia care (MAC) and involves variable sedation, analgesia, and anxiolytics as needed. The choice of anesthesia depends on patient-related factors and area-specific practices. The clinical outcomes are similar between the two approaches (general anesthesia and MAC); however, the MAC approach is associated with shorter hospital stays [21].

\section{Percutaneous access}

Previously, TAVI was performed with surgical cutdown at the access site, but with advances in medical technology, the percutaneous approach is used with delivery sheaths as small as 14-16 French [9]. The puncture site is visualized by ultrasound or fluoroscopic angiography [22]. Finally, percutaneous closure should be performed as it is minimally invasive and provides for a shorter hospital stay as compared with suture-based closure [23]. The devices available for percutaneous closure are Prostar (Abbott, Abbott Park, IL), ProGlide (Abbott), and MANTA (Teleflex, Morrisville, NC) [24].

\section{Valve implantation}

Initially, balloon valvuloplasty was used as a means of pre-dilatation before valve implantation but has been found to increase the risk of cerebral embolization and severe acute aortic regurgitation [25]. Therefore, it is currently performed in only complex cases of severely calcified aortic valves. As we have improved delivery systems and prosthesis, balloon valvuloplasty can safely be avoided to reduce procedure time and complications [26]. After puncturing the site, a long catheter is inserted over the sheath followed by the aortography to guide valve placement. A Safari or Amplatz Super Stiff guidewire (Boston Scientific, Marlborough, MA) is introduced into the LV, which assists in the deployment of an Edwards SAPIEN or Medtronic valve at the site of the aortic valve. The temporary pacemaker is attached with a Safari wire to test for rapid pacing, during which the valve expands and secures along the native aortic valve annulus. The temporary pacemaker is placed in the right ventricle only if the patient develops atrioventricular block or prolonged QRS duration after the procedure [27].

\section{Post-TAVI management}

Patients are monitored for hemodynamic stability and cardiac rhythm for 12 to 24 hours in the ICU before transferring to the critical care unit [26]. However, ICU admission can be omitted if proper pre- and postprocedure evaluations are completed [28]. Early discharge within 24 to 48 hours can be considered as it has no difference in mortality, stroke, and readmission as compared with late discharge after 48 hours [26]. This approach will also reduce the overall cost and staff workload. The patients are given heparin during the procedure, and a combination of aspirin and clopidogrel for six months post-procedure [29].

\section{Efficacy of TAVI versus SAVR}

There are several randomized controlled trials and large registries that studied the efficacy of different valves used in TAVI. Medtronic CoreValve, Edwards SAPIEN, and LOTUS Valve (Boston Scientific) are the available 
options. The safety of these valves was established by the PARTNER, CoreValve Pivotal, and RESPOND (Rivastigmine to Stabilise Gait in Parkinson's Disease) trials, respectively. Initially, the PARTNER trials discovered the efficacy of the Edwards SAPIEN valve by using two cohort populations. The PARTNER A trial compared the Edwards SAPIEN valve with SAVR in high surgical risk patients, and the PARTNER B cohort compared the Edwards SAPIEN valve with medical therapy in nonsurgical patients. The PARTNER A trial reported one-year mortality of $24.2 \%$ in the TAVI group and $26.8 \%$ in the SAVR group $(\mathrm{p}=0.44)$. The twoyear mortality was $33.9 \%$ in the TAVI group and $35 \%$ in the SAVR group ( $\mathrm{p}=0.78)$. The rate of stroke and transient ischemic attack (TIA) was $8.3 \%$ in the TAVI group and $4.3 \%$ in the SAVR group $(\mathrm{p}=0.04)$ at one year. The rate of major vascular complications was $11.0 \%$ with TAVI and $3.2 \%$ with SAVR. The major bleeding episodes were $9.3 \%$ in TAVI and $19.5 \%$ in SAVR, and atrial fibrillation was reported as $8.6 \%$ in TAVI and $16.0 \%$ in SAVR. The other cohort, in the PARTNER B trial, reported two-year mortality of $43.4 \%$ with TAVI versus $68.0 \%$ with medical therapy. The stroke rate at two years was $13.8 \%$ with TAVI but only $5.5 \%$ with medical therapy [9].

On the other hand, the CoreValve Pivotal trial used the self-expandable transcatheter valve and compared it with medical and surgical treatment. The mortality rate at one year was $14.1 \%$ in the TAVI group, whereas it was $18.9 \%$ in the SAVR group. The CoreValve Extreme Risk US Pivotal Trial reported a $26 \%$ rate of major stroke and all-cause mortality at one year as compared with $43 \%$ with a pre-specified estimate with medical therapy $[9,30]$. Another trial, RESPOND, evaluated outcomes of TAVI with the LOTUS Valve. The rate of mortality and stroke at 30 days was $2.6 \%$ and $3.3 \%$, respectively. It was effective in increasing the aortic valve area from $0.7 \mathrm{~cm} 2$ at baseline to $1.8 \mathrm{~cm} 2$ at discharge [31].

Hence, with the use of the Edwards SAPIEN, the mortality rate was comparably the same in both the TAVI and SAVR groups. There was an increased risk of stroke, TIA, paravalvular regurgitation, and major vascular complications with TAVI, whereas more cases of major bleeding and atrial fibrillation were reported in the SAVR group. The mortality rate and repeat hospitalization were lower with TAVI as compared with medical management. However, the rate of stroke remained high in the TAVI group. The use of the Medtronic CoreValve showed lower mortality at two years as compared with SAVR. It also demonstrated low rates of all-cause mortality and major stroke as compared with medical therapy. The results with the LOTUS Valve demonstrate they are also safe for implementing in clinical practice. The major adverse effects of the TAVR and SAVR are shown in Figure 1.

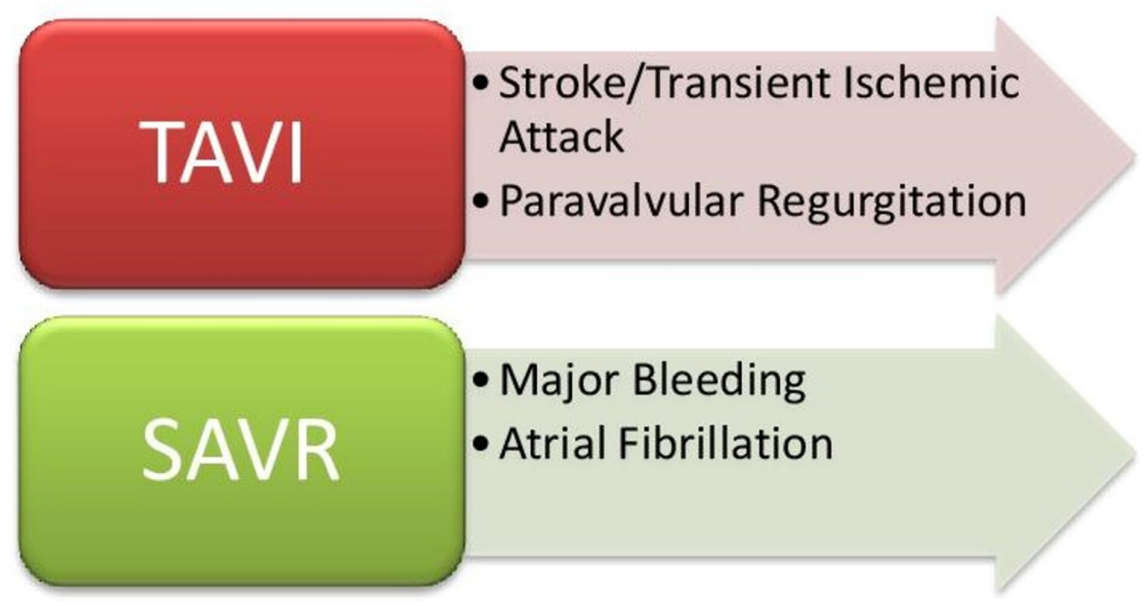

FIGURE 1: Complications of TAVI and SAVR

TAVI, transcatheter aortic valve implantation; SAVR, surgical aortic valve replacement

\section{Follow-up monitoring}

Post-procedural echocardiography should be performed before patient discharge, within 30 days of the procedure, after one year, and annually later on. Other modes of imaging can be used, if necessary. The long-run follow-up is pivotal in monitoring valve function and durability. Along with that, patients should also be clinically monitored for symptoms of congestive cardiac failure, infection, and embolism [32].

\section{Complications}

The procedure can have a wide array of peri-procedural and long-term complications. These range from bleeding, annular rupture, stroke, cardiac, and renal ischemia. There is a high chance of valvular malposition/dysfunction. The mechanical prosthesis requires lifelong anticoagulation and has high chances of lifetime bleeding and reoperation. The valvular bioprosthesis can have structural or nonstructural 
deterioration. Irreversible structural damage is due to tissue degeneration and proliferation, leading to intrinsic changes in the valve, tear, calcification, or pannus formation. Nonstructural valve deterioration is due to paravalvular regurgitation, prosthesis-patient mismatch, malposition, and embolization. Other complications can be thrombosis and endocarditis [32].

\section{Differences in mortality of TAVI versus SAVR}

The graph in Figure 2 compares mortality due to SVAR and TAVI in four different randomized clinical trials. The graph assesses the primary outcome of all-cause mortality at the two-year follow-up.

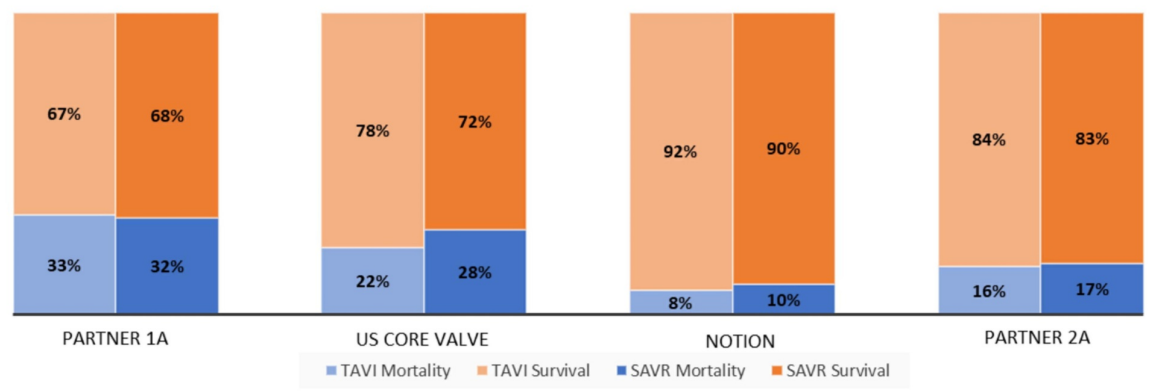

FIGURE 2: This graph compares the mortality in the TAVI group versus the SAVR group in four different randomized clinical trials. These trials assess the primary outcomes of all-cause mortality at two-year followup

TAVI, transcatheter aortic valve implantation; SAVR, surgical aortic valve replacement

\section{Future implications}

TAVI, with a minimalistic approach, has been increasingly used in many centers. With increasing procedural experience, improvements in patient selection, and newer device performance, the rate of mortality and complications will drop. More modern devices continue to offer promising features such as proven durability, small delivery catheter, better positioning, and retrieval mechanism [31]. These improved traits will markedly shift the conventional practices over time.

\section{Gaps in knowledge}

The quest for identifying the optimal method for the management of AS has just started, and there are several gaps in our knowledge that need to be investigated. Studies should investigate the efficacy of elective SAVR in asymptomatic patients with severe AS. The criteria for choice between TAVI and SAVR must be further established in low-operative risk patients. Studies should be conducted to evaluate the factors responsible for conduction disturbances to minimize pacemaker implantations [33]. Furthermore, patients who undergo TAVI should be studied long term (i.e., beyond five years) to identify complications and evaluate the durability of the valves.

\section{Conclusions}

TAVI is a minimally invasive intervention to treat severe symptomatic AS as compared with SAVI. TAVI has better mortality outcomes as compared with SAVR. However, patients who undergo TAVI are at a high risk of major vascular events such as stroke; therefore, a risk-benefit assessment should be performed before going forward with an intervention of TAVI or SAVR for AS.

\section{Additional Information}

\section{Disclosures}

Conflicts of interest: In compliance with the ICMJE uniform disclosure form, all authors declare the following: Payment/services info: All authors have declared that no financial support was received from any organization for the submitted work. Financial relationships: All authors have declared that they have no financial relationships at present or within the previous three years with any organizations that might have an interest in the submitted work. Other relationships: All authors have declared that there are no other relationships or activities that could appear to have influenced the submitted work.

\section{References}


1. Coffey S, Cox B, Williams MJA: The prevalence, incidence, progression, and risks of aortic valve sclerosis . J Am Coll Cardiol. 2014, 63:2852-61. 10.1016/j.jacc.2014.04.018

2. Osnabrugge RLJ, Mylotte D, Head SJ, et al.: Aortic stenosis in the elderly: disease prevalence and number of candidates for transcatheter aortic valve replacement: a meta-analysis and modeling study. J Am Coll Cardiol. 2013, 62:1002-12. 10.1016/j.jacc.2013.05.015

3. Clark MA, Duhay FG, Thompson AK, et al.: Clinical and economic outcomes after surgical aortic valve replacement in Medicare patients. Risk Manag Healthc Policy. 2012, 117:117-26. Accessed: December 19, 2019: 10.2147/RMHP.S34587

4. Baumgartner H, Falk V, Bax JJ, et al.: 2017 ESC/EACTS guidelines for the management of valvular heart disease. Eur Heart J. 2017, 38:2739-91. 10.1093/eurheartj/ehx391

5. Barasch E, Fan D, Chukwu EO, et al.: Severe isolated aortic stenosis with normal left ventricular systolic function and low transvalvular gradients: pathophysiologic and prognostic insights. J Heart Valve Dis. 2008, $17: 81-8$.

6. Minners J, Allgeier M, Gohlke-Baerwolf C, Kienzle RP, Neumann FJ, Jander N: Inconsistent grading of aortic valve stenosis by current guidelines: haemodynamic studies in patients with apparently normal left ventricular function. Heart. 2010, 96:1463-8. 10.1136/hrt.2009.181982

7. Belkin RN, Khalique O, Aronow WS, Ahn C, Sharma M: Outcomes and survival with aortic valve replacement compared with medical therapy in patients with low-, moderate-, and severe-gradient severe aortic stenosis and normal left ventricular ejection fraction. Echocardiography. 2011, 28:378-87. 10.1111/j.15408175.2010.01372.x

8. Marquis-Gravel G, Redfors B, Leon MB, Généreux P: Medical treatment of aortic stenosis. Circulation. 2016, 134:1766-84. 10.1161/CIRCULATIONAHA.116.023997

9. Zeeshan A, Tuzcu EM, Krishnaswamy A, Kapadia S, Mick S: Transcatheter aortic valve replacement: history and current indications. Cleve Clin J Med. 2015, 82:6-10. 10.3949/ccjm.82.s2.02

10. Baumgartner H: Aortic stenosis: medical and surgical management. Heart. 2005, 91:1483-8. 10.1136/hrt.2004.056176

11. Moreno PR, Jang IK, Newell JB, Block PC, Palacios IF: The role of percutaneous aortic balloon valvuloplasty in patients with cardiogenic shock and critical aortic stenosis. J Am Coll Cardiol. 1994, 23:1071-5. 10.1016/0735-1097(94)90592-4

12. Mariathas M, Rawlins J, Curzen N: Transcatheter aortic valve implantation: where are we now? . Future Cardiol. 2017, 13:551-66. 10.2217/fca-2017-0056

13. Rashedi N, Otto CM: Aortic stenosis: changing disease concepts. J Cardiovasc Ultrasound. 2015, 23:59-69. 10.4250/jcu.2015.23.2.59

14. Stewart RAH, Kerr AJ, Whalley GA, et al.: Left ventricular systolic and diastolic function assessed by tissue Doppler imaging and outcome in asymptomatic aortic stenosis. Eur Heart J. 2010, 31:2216-22. 10.1093/eurheartj/ehq159

15. Kang DH, Park SJ, Lee SA, et al.: Early surgery or conservative care for asymptomatic aortic stenosis. N Engl J Med. 2020, 382:10-1056. 10.1056/NEJMoa1912846

16. Al-Adhami A, Al-Attar N: Recent advances in aortic valve replacement for aortic stenosis . F1000Res. 2016, 5:2542. Accessed: December 19, 2019: 10.12688/f1000research.8728.1

17. Chieffo A, Giustino G, Spagnolo P, et al.: Routine screening of coronary artery disease with computed tomographic coronary angiography in place of invasive coronary angiography in patients undergoing transcatheter aortic valve replacement. Circ Cardiovasc Interv. 2015, 8:002025. 10.1161/CIRCINTERVENTIONS.114.002025

18. Moat NE, Ludman P, de Belder MA, et al.: Long-term outcomes after transcatheter aortic valve implantation in high-risk patients with severe aortic stenosis. J Am Coll Cardiol. 2011, 58:2130-8. 10.1016/j.jacc.2011.08.050

19. Otto CM, Kumbhani DJ, Alexander KP, et al.: 2017 ACC expert consensus decision pathway for transcatheter aortic valve replacement in the management of adults with aortic stenosis. J Am Coll Cardiol. 2017, 69:131346. 10.1016/j.jacc.2016.12.006

20. Brecker SJ, Bleiziffer S, Bosmans J, et al.: Impact of anesthesia type on outcomes of transcatheter aortic valve implantation (from the Multicenter ADVANCE Study). Am J Cardiol. 2016, 117:1332-8. 10.1016/j.amjcard.2016.01.027

21. Huang J, Wang S, Lu J: Awake transcatheter aortic valve replacement-an anesthesiologist's perspective . J Vis Surg. 2018, 4:44. Accessed: December 19, 2019: 10.21037/jovs.2018.01.17

22. van Wiechen MP, Ligthart JM, Van Mieghem NM: Large-bore vascular closure: new devices and techniques . Interv Cardiol. 2019, 14:17-21. 10.15420/icr.2018.36.1

23. Kadakia MB, Herrmann HC, Desai ND, et al.: Factors associated with vascular complications in patients undergoing balloon-expandable transfemoral transcatheter aortic valve replacement via open versus percutaneous approaches. Circ Cardiovasc Interv. 2014, 7:570-6. 10.1161/CIRCINTERVENTIONS.113.001030

24. Barbash IM, Barbanti M, Webb J, et al.: Comparison of vascular closure devices for access site closure after transfemoral aortic valve implantation. Eur Heart J. 2015, 36:3370-9. 10.1093/eurheartj/ehv417

25. Grube E, Naber C, Abizaid A, et al.: Feasibility of transcatheter aortic valve implantation without balloon pre-dilation. JACC Cardiovasc Interv. 2011, 4:751-7. 10.1016/j.jcin.2011.03.015

26. Akodad M, Lefèvre T: TAVI: simplification is the ultimate sophistication . Front Cardiovasc Med. 2018, 5:96. Accessed: December 19, 2019: 10.3389/fcvm.2018.00096

27. Sawaya FJ, Lefèvre T, Spaziano M, et al.: Transfemoral transcatheter aortic valve implantation: how minimalistic can we become?. J Interv Cardiol. 2016, 29:628-31. 10.1111/joic.12348

28. Leclercq F, Iemmi A, Lattuca B, et al.: Feasibility and safety of transcatheter aortic valve implantation performed without intensive care unit admission. Am J Cardiol. 2016, 118:99-106. 10.1016/j.amjcard.2016.04.019

29. Leon MB, Smith CR, Mack M, et al.: Transcatheter aortic-valve implantation for aortic stenosis in patients who cannot undergo surgery. N Engl J Med. 2010, 363:1597-607. 10.1056/NEJMoa1008232

30. Siontis GCM, Praz F, Pilgrim T, et al.: Transcatheter aortic valve implantation vs. surgical aortic valve 


\section{Cureus}

replacement for treatment of severe aortic stenosis: a meta-analysis of randomized trials. Eur Heart J. 2016, 37:3503-12. 10.1093/eurheartj/ehw225

31. Lüscher TF: Managing aortic stenosis with TAVI or surgery: risk assessement and long-term outcome . Eur Heart J. 2017, 38:3327-9. 10.1093/eurheartj/ehx681

32. Eltchaninoff H, Durand E, Barbanti M, Abdel-Wahab M: TAVI and valve performance: update on definitions, durability, transcatheter heart valve failure modes and management. EuroIntervention. 2018, 14:64-73. 10.4244/EIJ-D-18-00653

33. Mangieri A, Montalto C, Pagnesi M, et al.: TAVI and post procedural cardiac conduction abnormalities . Front Cardiovasc Med. 2018, 5:85. Accessed: December 19, 2019: 10.3389/fcvm.2018.00085 